\title{
A micromachined device describing over a hundred orders of parametric resonance
}

\author{
Yu Jia, ${ }^{1, a)}$ Sijun Du, ${ }^{1}$ Emmanuelle Arroyo, ${ }^{1}$ and Ashwin A. Seshia ${ }^{1}$ \\ Nanoscience Centre, Department of Engineering, University of Cambridge, 11 JJ Thomson Avenue, Cambridge, \\ CBз OFF, UK.
}

(Dated: 10 April 2018)

Parametric resonance in mechanical oscillators can onset from the periodic modulation of at least one of the system parameters, and the behaviour of the principal (1st order) parametric resonance has long been well established. However, the theoretically predicted higher orders of parametric resonance, in excess of the first few orders, have mostly been experimentally elusive due to the fast diminishing instability intervals. A recent paper experimentally reported up to 28 orders in a micromachined membrane oscillator. This paper reports the design and characterisation of a micromachined membrane oscillator with a segmented proof mass topology, in an attempt to amplify the inherent nonlinearities within the membrane layer. The resultant oscillator device exhibited up to over a hundred orders of parametric resonance, thus experimentally validating these ultra high orders as well as overlapping instability transitions between these higher orders. This research introduces design possibilities for the transducers and dynamics communities, by exploiting the behaviour of these previously elusive higher order resonant regimes.

PACS numbers: 77.65.Fs, 77.65.Ly, 87.19.ln

Keywords: Parametric resonance, Transducer, MEMS

\section{INTRODUCTION}

Parametric resonance is a nonlinear vibratory phenomenon that onsets when at least one of the system parameters are periodically perturbed and the system operates within one of the instability regimes. Unlike directly forced systems, the response frequency of a parametrically excited system is not directly linked to the external excitation, but instead matches the natural frequency. This intriguing vibratory phenomenon dates back to an observation reported by Michael Faraday in 1831루, subsequently experimentally verified by Lord Rayleigh (J. Strutt) in the 1880 's ${ }^{213}$ and its mathematical formulation established by Mathieu ${ }^{4}$, Floquet ${ }^{5}$ and Hill ${ }^{6}$. A parametric oscillator, in the most basic form, can be described by the generic Mathieu function shown in equation 1 .

$$
\ddot{x}+[\delta-2 \varepsilon \cos (2 t)] x=0
$$

where, $x$ is the oscillatory displacement, $\delta$ relates to an arbitrary frequency squared parameter, $\epsilon$ represents an arbitrary parametric excitation amplitude, and $t$ is the time domain.

While the characteristics of the first order (principal) parametric resonance has long been well established and its instability Strutt chart characteristics has been comprehensively experimentally validated 78 , the behaviour of higher order parametric resonances has received relatively less attention in the research community. Experimentally, it is significantly more difficult to observe

\footnotetext{
a)Electronic mail: yu.jia.gb@ieee.org also at Department of Mechanical Engineering, Thornton Science Park, University of Chester, Chester, CH2 4NU, UK.
}

and study the higher orders due to the increasingly rapid diminishing instability intervals at higher orders 9 , for a damped Mathieu type system. Turner et al!10 and Jia et al ${ }^{11}$ observed up to 5 orders of parametric resonance in MEMS oscillators, while $\mathrm{Yu}$ et al ${ }^{12}$ observed up to 4 orders of parametric resonance in a nanowire oscillator. More recently, Jia et al $\frac{13}{}$ reported up to 28 orders using a MEMS disk membrane with a central mass. It showed preliminary evidence that instability intervals at some higher orders start to overlap, despite the rapid increase in stability gap. Such a behaviour, while predicted in theory, has not been reported in experiment. Therefore, the overlap and the instability transitions at higher orders are experimental behaviour that warrant further investigation. This paper builds on to previous work by designing and testing a MEMS membrane topology that has segmented proof mass in an attempt to maximise nonlinearity.

The applications of parametric resonance have potential implications for the transducers research and development. The phenomenon has already been implemented as a mechanical amplifier for enhancing the sensitivity of MEMS vibratory sensors such as gyroscopes ${ }^{14}$ and magnetometers ${ }^{15}$, as well as improving the power density generated by vibration energy harvesters at both macroscale ${ }^{16} 17$ and in MEMS devices ${ }^{18}$. The experimental investigation of ultra high orders of parametric resonance, thus opens up avenues of design to exploit these previously elusive resonant regimes for further applications.

\section{THEORY AND SIMULATION}

It has previously been shown that Duffing nonlinearity can lead to small perturbations described by the Mathieu 
function 13 . The residual stress on the piezoelectric (AlN) MEMS device can be a source of inherent geometric nonlinearity. This is specifically apparent for nitride based layer on a silicon layer, due to the distinct thermal expansion coefficients. Equation 2 describes a parametric oscillator with nonlinear damping and stiffness $\frac{16}{}$.

$$
\ddot{x}+2 \epsilon \zeta_{1} \omega_{0} \dot{x}+\zeta_{2} \dot{x}|\dot{x}|+\left[\omega_{0}^{2}+\epsilon \omega^{2} \alpha \cos (\omega t)\right] x+\mu x^{3}=0
$$

where, $\epsilon$ is the bookmark term $\frac{19}{19}$ used to numerically study the function, $\zeta_{1}$ is linear damping ratio, $\zeta_{2}$ is nonlinear damping ratio, $\omega$ is the excitation frequency, $\omega_{0}$ is the natural frequency as well as the parametric resonant response frequency, $\alpha$ is the parametric excitation amplitude, and $\mu$ is the mass normalised third order nonlinear spring stiffness coefficient.

The instability region can be accessed when $\omega$ is in the vicinity of $2 \omega_{0} / n$; where, $n$ is the order number of parametric resonance as well as the interval number between each Mathieu instability zones in the Strutt instability diagram (figure 11). At higher orders, the instability regions become narrower at small amplitudes. However, at larger amplitudes, the overlap between neighbouring instability regions also become more substantiated.

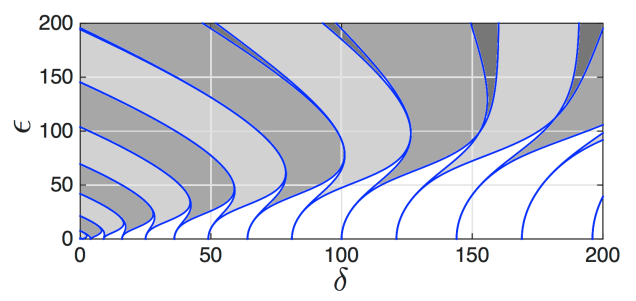

FIG. 1: Strutt instability diagram of first 14 orders.

Blue lines traces out the loci separating the unstable (greyed area) and the stable zones. Mild grey represents the odd orders, medium grey represents the even orders,

and dark grey illustrates the overlapping transitional

regions between neighbouring instability zones.

For $n=101$, an example of the time domain and phase plane response can be seen in figure 2. In both scenarios, the values for excitation frequency $\omega$ and amplitude $\alpha$ were fixed, while varying $\mu$ values were explored.

Figure 3 shows the time domain response of equation 2 for $n=1$. It can be seen that the response frequency (near the natural frequency) is at half the frequency of the excitation.

\section{DESIGN AND METHOD}

The deign of the proposed MEMS topology can be seen in figure 4. The central mass of the disk membrane is segmented along various arcs. Therefore, in addition to the circular membrane sections, the lines and core between the segmented mass also experience significant stress.
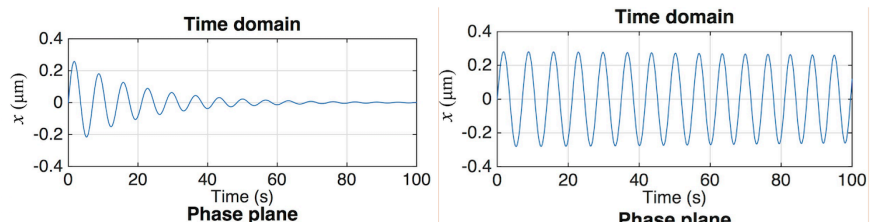

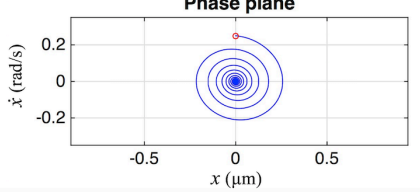

(a) Within stable region

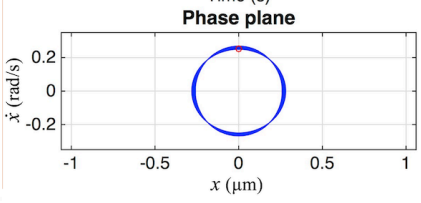

(b) Within instability region
FIG. 2: Simulated response near the vicinity of $n=101$. (a) represents non-resonant response at low $\mu$ (b) illustrates parametric resonance at high $\mu$.

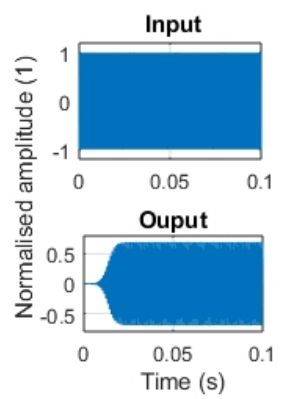

(a) $\mathrm{t}=0 \mathrm{~s}$ to $0.1 \mathrm{~s}$

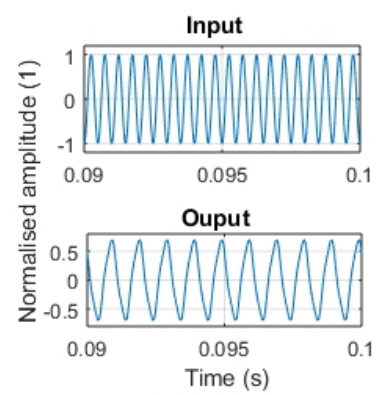

(b) $\mathrm{t}=0.09 \mathrm{~s}$ to $0.1 \mathrm{~s}$
FIG. 3: Time domain of parametric oscillation with parametric nonlinear stiffness. Output $\left(\right.$ near $\left.\omega_{0}\right)$ is at half the frequency of the input $\left(2 \omega_{0}\right)$.
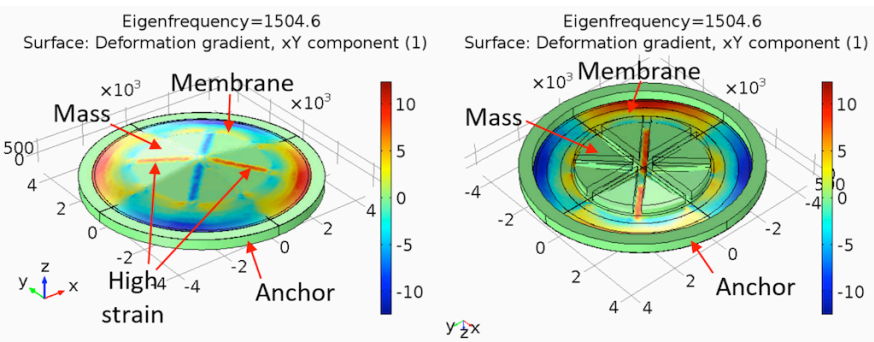

FIG. 4: FE simulated first mode shape at $f_{1}=1505 \mathrm{~Hz}$ (equivalent to the 0,1 circular membrane mode), showing top view (left) and back view (right).

Comparing with a previously reported plain membrane topology with a centered mass $\frac{13}{13}$, the segmented mass topology aims to intentionally accumulate more residual stress. The MEMS devices were fabricated using an AlNon-SOI process. A $0.5 \mu \mathrm{m}$ AlN piezoelectric layer is used as the transducer of the MEMS oscillator, a $1 \mu \mathrm{m}$ Al layer is used as the top electrode and bond pads, while a 10 $\mu \mathrm{m}$ doped silicon layer acts both as the bottom electrode as well as the device layer. Further details of the process is given in the supplementary document.

View of a fabricated device can be seen in figure 5 . 
The net membrane radius between the anchors is $4 \mathrm{~mm}$, while the effective radius of the central mass is $2.5 \mathrm{~mm}$. Mass segmentations of $300 \mu \mathrm{m}$ width section the centred mass into 8 equal sized circular sectors. Two $500 \mu \mathrm{m}$ wide circular electrode rings are placed on the circular membrane surface to route out the electrical signals.

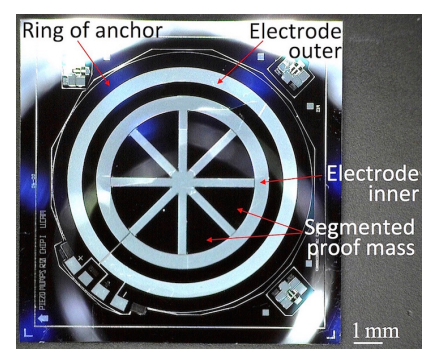

(a) Top view

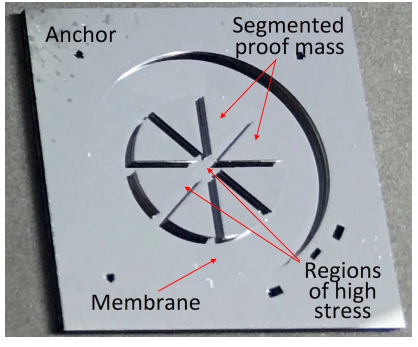

(b) Back view
FIG. 5: Fabricated MEMS oscillator device. The silicon die is $11 \mathrm{~mm}$ by $11 \mathrm{~mm}$ in dimensions.

The MEMS dies were integrated with leadless chip carriers and measurement board. The devices were experimentally characterised using a mechanical shaker setup controlled by a digital function generator and measured using a digital oscilloscope. The experimental method is described further in the supplementary document.

\section{RESULTS AND DISCUSSION}

As variations due to fabrication tolerances exist across the same batch of fabricated devices, different dies exhibited slightly different natural frequencies and the number of orders of parametric resonance observable. One device recorded up to 101 orders of confirmed parametric resonance (figure 6).

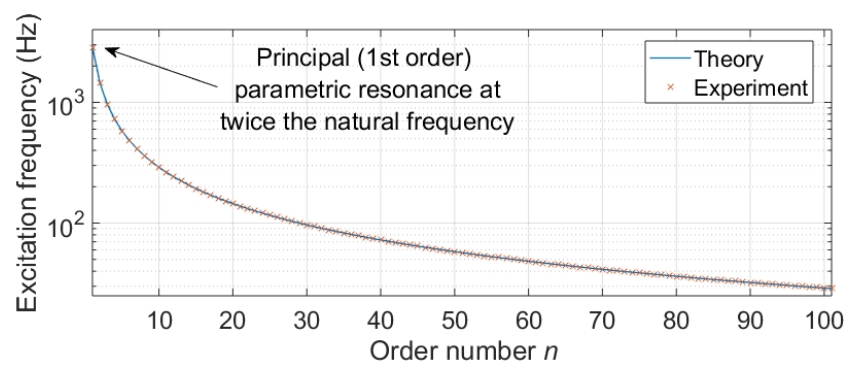

FIG. 6: Excitation frequency values of the resonant peaks of $1 \leq n \leq 101$ orders of parametric resonance.

While parametric oscillatory response may still exist beyond $n=101$ for this specific MEMS oscillator, it was not experimentally feasible to verify the higher order responses as the signal amplitude was embedded within the inherent noise floor. A key verification step was to compare the input and output frequencies relative to the $\omega: 2 \omega_{0} / n$ ratio 8 . For instance, at $n=1$, the input frequency was $2900 \mathrm{~Hz}$ and the response frequency was $1450 \mathrm{~Hz}$; and at $n=101$, the input frequency $28.7 \mathrm{~Hz}$, while the response frequency was also $1450 \mathrm{~Hz}$.

Figure 7 presents the frequency domain characteristics of the parametric oscillator. Duffing behaviour of the oscillator driven into direct resonance can also be seen. Despite sharing the same frequency vicinity, $n=2$ does not superimpose with direct resonance; and only one of the two resonant responses can exist.

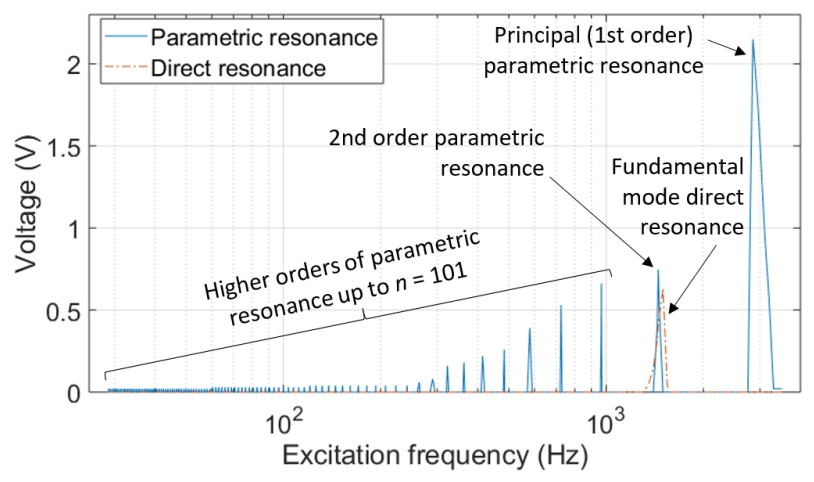

FIG. 7: Frequency domain response of the oscillator when excited at $1 \mathrm{~g}$ acceleration amplitude.

At higher orders, the instability regions diminishes in both amplitude response and frequency bandwidth as theoretically predicted. This points to the historic difficulty in conducting practical measurements in built mechanical systems. For MEMS oscillators with high natural frequencies, the absolute bandwidth is relatively wider and can be more readily measured. Table $\mathrm{I}$ lists the frequencies of a few selected higher orders.

TABLE I: Frequency values for selected higher orders.

\begin{tabular}{c|c}
\hline Order number $n$ & Excitation frequency $\omega(\mathrm{Hz})$ \\
\hline 95 & 30.5 \\
96 & 30.2 \\
97 & 29.9 \\
98 & 29.6 \\
99 & 29.3 \\
100 & 29.0 \\
101 & 28.7 \\
\hline
\end{tabular}

Figure 8 illustrates the overlapping nature of the instability regions near these high orders. When sweeping downwards, the activated instability region extends into the vicinity of the next order. For all higher orders (in excess of $\sim 30$ ), a qualitatively similar overlapping transition has been observed. However, there is a degree of experimental uncertainty of at which specific order the overlapping transition onsets.

While theoretically the overlapping transition of instability regions is predicted as shown in figure 1 this has been difficult to experimentally verify thus far. One of the main reasons is because the overlapping transitions only emerges to become more significant relative to the 
rest of the instability regions at higher orders. Though, within the practical confines of this experiment, an additional reason for observing an apparent overlapping behaviour could be attributed to the limited frequency resolution and purity of the excitation signal provided by the mechanical shaker, thus adding to the uncertainty of when a genuine instability overlap onsets. However, this also implies that in practical applications where external excitations lack infinitesimal resolution, such an overlap could manifest.

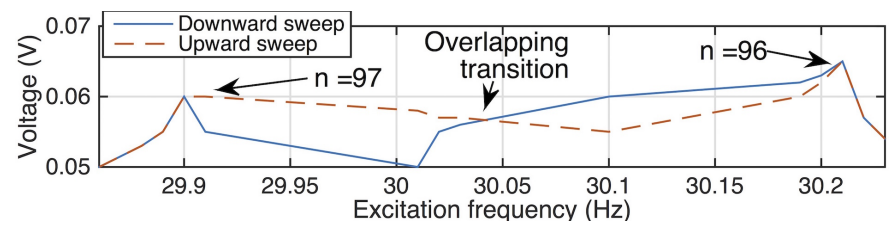

FIG. 8: Frequency domain response showing overlapping instability transition between orders $n=96$ and $n=97$.

The investigation of the instability transition can prove valuable, as the frequency at which the transition takes place is extremely sensitive to boundary conditions. Therefore, by charting the loci of the ultra high order parametric resonant instability regions, any variations in external influences can be mapped. Therefore, analogous to the frequency-dependent resonant MEMS sensors 20 , a potential instability transition dependent sensing mechanism can potentially be designed 21 .

\section{CONCLUSION}

This paper reports a segmented mass membrane topology for MEMS parametric oscillators. The oscillator utilises inherently high geometric nonlinearity from the proposed design in order to promote the Duffing-Mathieu link. Up to 101 orders of parametric resonance were experimentally observed, along with overlapping transitional regions between the higher order instability zones. This opens up the possibility to investigate the behaviour of these ultra high orders and exploit these previously elusive resonant regimes for transducer and oscillator research.

\section{SUPPLEMENTARY MATERIAL}

Please see the accompanying supplementary document for the details of the MEMS fabrication process, experimental method, measured data, and postulation of possible hypotheses in discussion of interpreting the observed experimental results.

\section{ACKNOWLEDGEMENT}

This research was supported by funding from EPSRC (EP/L010917/1).

\section{REFERENCES}

${ }^{1}$ M. Faraday, Phil. Trans. , 299 (1831).

${ }^{2}$ J. Strutt, Philos. Mag. 15, 229 (1883).

${ }^{3}$ J. Strutt, Philos. Mag. 24, 145 (1887).

${ }^{4}$ M. Mathieu, J. Math. Pures Appl. 13, 137 (1868).

${ }^{5}$ G. Floquet, Ann. Sci. Ec. Norm. Sup. 12, 47 (1883).

${ }^{6}$ G. Hill, Acta Math. 8, 1 (1886).

${ }^{7}$ M. Cartmell, J. Sound Vib. 143, 395 (1990).

${ }^{8}$ A. Tondl, T. Ruijgrok, F. Verhulst, and R. Nabergoj, Autoparametric resonance in mechancial systems (Cambridge University Press, Cambridge, UK, 2000).

${ }^{9}$ D. Levy and J. Keller, Commun. Pure Appl. Math. 16, 469 (1963).

${ }^{10}$ K. Turner, S. Miller, P. Hartwell, N. MacDonald, S. Strogatz, and S.G.Adams, Nature 396, 149 (1988).

${ }^{11}$ Y. Jia, J. Yan, K. Soga, and A. Seshia, in J. Phys. Conf. Ser., 1, Vol. 476 (2013) pp. pp. 607-611.

${ }^{12} \mathrm{M}$. Yu, G. Wagner, R. Ruoff, and M. Dyer, Phys. Rev. B 22, 073406 (2002).

${ }^{13}$ Y. Jia, S. Du, and A. Seshia, Scientific Reports 6 (2016).

${ }^{14} \mathrm{Y}$. Lee, A study of parametric excitation applied to a MEMS tuning fork gyroscope, Ph.D. thesis, University of Missouri (2007).

${ }^{15} \mathrm{M}$. Thompson and D. Horsely, in Proc. Transducers 2009 (Denvor, CO, 2009) pp. 1194-1197.

${ }^{16}$ M. Daqaq, C. Stabler, Y. Qaroush, and T. Seuaciuc-Osorio, J. Intel. Mat. Syst. Str. 20, 545 (2009).

${ }^{17}$ Y. Jia, J. Yan, K. Soga, and A. Seshia, J. Intel. Mat. Syst. Str. 25 (2014).

${ }^{18}$ Y. Jia, J. Yan, K. Soga, and A. Seshia, J. Micromech. Microeng 23, 10pp. (2013).

${ }^{19}$ B. Zaghari, E. Rustighi, and M. G. Tehrani, Smart Materials and Nondestructive Evaluation for Energy Systems 9439 (2015).

${ }^{20}$ C. D. Do, A. Erbes, J. Yan, K. Soga, and A. A. Seshia, J. Microelectromech. Syst. 25, 851 (2016).

${ }^{21}$ L. L. Li, E. L. Holthoff, L. A. Shaw, C. B. Burgner, and K. L. Turner, J. Microelectromech. Syst. 23, 1228 (2014). 